\title{
Stage IB Bone Cancer AJCC v8
}

National Cancer Institute

\section{Source}

National Cancer Institute. Stage IB Bone Cancer A/CC v8. NCI Thesaurus. Code C136615.

Stage IB includes: (T2, N0, M0, G1 or GX); (T3, N0, M0, G1 or GX). T2: Tumor measuring more than $8 \mathrm{~cm}$ in greatest dimension. T3: Discontinuous tumors in the primary bone site. N0: No regional lymph node metastasis. M0: No distant metastasis. GX: Grade cannot be assessed. G1: Well differentiated, low grade. (AJCC 8th ed.) 